\title{
BOOLEAN DIMENSION AND LOCAL DIMENSION
}

\author{
WILLIAM T. TROTTER ${ }^{1}$ AND BARTOSZ WALCZAK ${ }^{2}$
}

\begin{abstract}
Dimension is a standard and well-studied measure of complexity of posets. Recent research has provided many new upper bounds on the dimension for various structurally restricted classes of posets. Bounded dimension gives a succinct representation of the poset, admitting constant response time for queries of the form "is $x<y$ ?". This application motivates looking for stronger notions of dimension, possibly leading to succinct representations for more general classes of posets. We focus on two: boolean dimension, introduced in the 1980s and revisited in recent research, and local dimension, a very new one. We determine precisely which values of dimension/boolean dimension/local dimension imply that the two other parameters are bounded.
\end{abstract}

\section{INTRODUCTION}

Dimension. The dimension of a poset $P=(X, \leqslant)$ is the minimum number of linear extensions of $\leqslant$ on $X$ the intersection of which gives $\leqslant$. More precisely, a realizer of a poset $P=(X, \leqslant)$ is a set $\left\{\leqslant_{1}, \ldots, \leqslant d\right\}$ of linear extensions of $\leqslant$ on $X$ such that

$$
x \leqslant y \Longleftrightarrow(x \leqslant 1 y) \wedge \cdots \wedge\left(x \leqslant \leqslant_{d} y\right), \quad \text { for any } x, y \in X,
$$

and the dimension is the minimum size of a realizer. The concept of dimension was introduced by Dushnik and Miller [8] and has been widely studied since. There are posets with arbitrarily large dimension: the standard example $S_{k}=\left(\left\{a_{1}, \ldots, a_{k}, b_{1}, \ldots, b_{k}\right\}, \leqslant\right)$, where $a_{1}, \ldots, a_{k}$ are minimal elements, $b_{1}, \ldots, b_{k}$ are maximal elements, and $a_{i}<b_{j}$ if and only if $i \neq j$, has dimension $k$ when $k \geqslant 2$ [8]. On the other hand, the dimension of a poset is at most the width [12], and it is at most $\frac{n}{2}$ when $n \geqslant 4$, where $n$ denotes the number of elements [12].

The cover graph of a poset $P=(X, \leqslant)$ is the graph on $X$ with edge set $\{x y: x<y$ and there is no $z$ with $x<z<y\}$. A poset is planar if its cover graph has a non-crossing upward drawing in the plane, which means that every cover graph edge $x y$ with $x<y$ is drawn as a curve that goes monotonically up from $x$ to $y$. Planar posets that contain a least element and a greatest element are well known to have dimension at most 2 [1]. By contrast, spherical posets (i.e. posets with upward non-crossing drawings on a sphere) with least and greatest elements can have arbitrarily large dimension [24]. Trotter and Moore [25] proved that planar posets that contain a least element have dimension at most 3 (and so do posets whose cover graphs are forests) and asked whether all planar posets have bounded dimension. The answer is no-Kelly [17] constructed planar posets with arbitrarily large dimension (pictured). Another property of Kelly's posets is that their cover graphs have path-width and treewidth 3. Recent research brought a plethora of new bounds on dimension for structurally restricted posets. In particular, dimension is bounded for

- posets with height 2 and planar cover graphs [9],

- posets with bounded height and planar cover graphs [23],

- posets with bounded height and cover graphs of bounded tree-width [14],

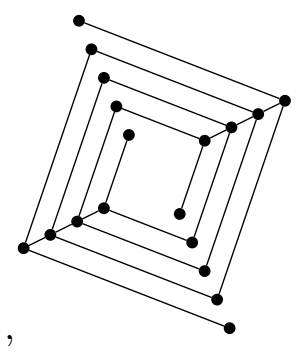

- posets with bounded height and cover graphs excluding a topological minor [27],

- posets with bounded height and cover graphs of bounded expansion [16],

\footnotetext{
${ }^{1}$ School of Mathematics, Georgia Institute of Technology, Atlanta, GA, USA, email: trotter@math.gatech.edu

${ }^{2}$ Department of Theoretical Computer Science, Faculty of Mathematics and Computer Science, Jagiellonian University, Kraków, Poland, email: walczak@tcs.uj.edu.pl

Bartosz Walczak was partially supported by National Science Center of Poland grant 2015/18/E/ST6/00299.
} 
- posets with cover graphs of path-width 2 [3],

- posets with cover graphs of tree-width 2 [15],

- posets with planar cover graphs excluding two incomparable chains of bounded length [13].

Boolean dimension. The boolean dimension of a poset $P=(X, \leqslant)$ is the minimum number of linear orders on $X$ a boolean combination of which gives $\leqslant$. More precisely, a boolean realizer of $P$ is a set $\left\{\leqslant_{1}, \ldots, \leqslant_{d}\right\}$ of linear orders on $X$ for which there is a $d$-ary boolean formula $\phi$ such that

$$
x \leqslant y \Longleftrightarrow \phi\left(\left(x \leqslant 1 \leqslant_{1} y\right), \ldots,(x \leqslant d y)\right) \quad \text { for any } x, y \in X,
$$

and the boolean dimension is the minimum size of a boolean realizer. The boolean dimension is at most the dimension, because a realizer is a boolean realizer for the formula $\phi\left(\alpha_{1}, \ldots, \alpha_{d}\right)=$ $\alpha_{1} \wedge \cdots \wedge \alpha_{d}$. Beware that the relation $\leqslant$ defined by (1) from arbitrary linear orders $\leqslant \leqslant_{1}, \ldots, \leqslant d$ on $X$ and formula $\phi$ is not necessarily a partial order.

Boolean dimension was first considered by Gambosi, Nešetřil, and Talamo [10] and by Nešetřil and Pudlák [22]. The definition above follows [22]. That in [10] allows only formulas $\phi$ of the form $\phi\left(\alpha_{1}, \ldots, \alpha_{d}\right)=\alpha_{i} \wedge \psi\left(\alpha_{1}, \ldots, \alpha_{i-1}, \alpha_{i+1}, \ldots, \alpha_{d}\right)$ for some $i$. The purpose of this restriction is unclear - it guarantees antisymmetry but not transitivity of the relation $\leqslant$ defined by (1). Under that modified definition, it is proved in [10] that boolean dimension $d$ and dimension $d$ are equivalent for $d \in\{1,2,3\}$ (we redo that proof in section 2 with no restriction on $\phi$ ). The standard examples $S_{k}$ with $k \geqslant 4$ have boolean dimension 4 [10] (see section 2). Easy counting shows that there are posets on $n$ elements with boolean dimension $\Theta(\log n)$ [22]. This is optimal - every $n$ element poset has boolean dimension $O(\log n)$ witnessed by a formula of length $O\left(n^{2} \log n\right)$ [22].

Nešetřil and Pudlák [22] asked whether boolean dimension is bounded for planar posets. It was proved already in [10] that posets with height 2 and planar cover graphs have bounded boolean dimension. Spherical posets with a least element also have bounded boolean dimension [6], contrary to ordinary dimension. This and the recent progress on dimension of structurally restricted posets have motivated revisiting boolean dimension in current research.

Local dimension. A partial linear extension of a partial order $\leqslant$ on $X$ is a linear extension of the restriction of $\leqslant$ to some subset of $X$. A local realizer of $P$ of width $d$ is a set $\left\{\leqslant_{1}, \ldots, \leqslant_{t}\right\}$ of partial linear extensions of $\leqslant$ such that every element of $X$ occurs in at most $d$ of $\leqslant 1, \ldots, \leqslant t$ and

$$
x \leqslant y \Longleftrightarrow \text { there is no } i \in\{1, \ldots, t\} \text { with } x>_{i} y, \quad \text { for any } x, y \in X .
$$

The local dimension of $P$ is the minimum width of a local realizer of $P$. Thus, instead of the size of a local realizer, we bound the number of times any element of $X$ occurs in it. A set of linear extensions of $\leqslant$ is a local realizer if and only if it is a realizer. In particular, the local dimension is at most the dimension. For arbitrary partial linear extensions $\leqslant 1, \ldots, \leqslant_{t}$ of $\leqslant$ on subsets of $X$, the relation $\leqslant$ defined by $(2)$ is not necessarily a partial order - it may fail to be antisymmetric or transitive. It is antisymmetric, for example, if one of $\leqslant_{1}, \ldots, \leqslant t$ is a linear extension of $\leqslant$ on $X$.

The concept of local dimension was proposed very recently by Ueckerdt [26] and originates from concepts studied in $[4,19]$. Ueckerdt $[26]$ also noticed that the standard examples $S_{k}$ with $k \geqslant 3$ have boolean dimension 3 .

Results. Extending the results on boolean dimension from [10], for each $d$, we determine whether posets with dimension/boolean dimension/local dimension $d$ have the other two parameters bounded or unbounded. Here is the full picture:

A. Boolean dimension $d$ and dimension $d$ are equivalent for $d \in\{1,2,3\}$ [10].

B. Local dimension $d$ and dimension $d$ are equivalent for $d \in\{1,2\}$.

C. The standard examples $S_{k}$ have boolean dimension 4 when $k \geqslant 4$ [10], local dimension 3 when $k \geqslant 3$ [26], and dimension $k$ when $k \geqslant 2$ [8].

D. There are posets with boolean dimension 4 and unbounded local dimension.

E. Posets with local dimension 3 have bounded boolean dimension.

F. There are posets with local dimension 4 and unbounded boolean dimension. 
We present proofs of $\mathrm{A}-\mathrm{F}$ in the next section.

Other new results concern boolean dimension and local dimension of structurally restricted posets. In particular, posets with cover graphs of bounded path-width have bounded boolean dimension [21] and bounded local dimension [2], while local dimension is unbounded for posets with cover graphs of tree-width $3[2,5]$ and for planar posets [5]. It remains open whether boolean dimension is bounded for posets with cover graphs of bounded tree-width (in particular, tree-width 3 ) and for planar posets. There are $n$-element posets with local dimension $\Theta(\sqrt{n})[2]$, and conceivably the right bound is $\left(\frac{1}{2}-o(1)\right) n$.

\section{Proofs}

A. Boolean dimension $d$ and dimension $d$ are equivalent for $d \in\{1,2,3\}$.

Proof. We basically repeat the argument given in [10] but avoiding the restriction on functions $\phi$ imposed therein. Let $P=(X, \leqslant)$ be a poset with boolean dimension $d$ and $\left\{\leqslant 1, \ldots, \leqslant_{d}\right\}$ be its boolean realizer for a formula $\phi$. Reflexivity of $\leqslant$ implies $\phi(1, \ldots, 1)=1$. Without loss of generality, assume $\phi(\alpha)=0$ when $\phi(\alpha)$ is never used by (1). This and antisymmetry of $\leqslant$ imply $\phi(\alpha)=0$ or $\phi(\bar{\alpha})=0$ for every $\alpha \in\{0,1\}^{d}$. In particular, $\phi(\alpha)=1$ for at most half of the tuples $\alpha$.

If $d=1$, then $\phi(1)=1$ and $\phi(0)=0$, so $\left\{\leqslant_{1}\right\}$ is a realizer of $P$. This shows that boolean dimension 1 and dimension 1 are equivalent.

Now, let $d \in\{2,3\}$. If $\phi(\alpha)=\phi(\bar{\alpha})=0$ for at most one pair $\alpha, \bar{\alpha}$ (as it is for $d=2$ ), then the strict partial order $\prec$ on $X$ defined by $x \prec y \Longleftrightarrow\left(\left(x<_{1} y\right), \ldots,\left(x<_{d} y\right)\right)=\alpha$ for distinct $x, y \in X$ is a transitive orientation of the incomparability graph of $P$, so $P$ has dimension at most 2 [8, Theorem 3.61]. This shows that boolean dimension 2 and dimension 2 are equivalent.

For $d=3$, to complete the proof that boolean dimension 3 and dimension 3 are equivalent, we consider the three cases (up to symmetry) in which $\phi(\alpha)=1$ for at most two tuples $\alpha$.

1. If $\phi(\alpha)=1$ for $\alpha=(1,1,1)$ only, then $\left\{\leqslant_{1}, \leqslant_{2}, \leqslant_{3}\right\}$ is a realizer of $P$.

2. If $\phi(\alpha)=1$ for $\alpha \in\{(1,1,0),(1,1,1)\}$ only, then $\left\{\leqslant_{1}, \leqslant_{2}\right\}$ is a realizer of $P$.

3. If $\phi(\alpha)=1$ for $\alpha \in\{(0,0,1),(1,1,1)\}$ only, then the strict partial order $\prec$ on $X$ defined by $x \prec y \Longleftrightarrow\left(\left(x<_{1} y\right) \wedge\left(x>_{2} y\right)\right)$ for distinct $x, y \in X$ is a transitive orientation of the incomparability graph of $P$, so $P$ has dimension at most 2 [8, Theorem 3.61].

B. Local dimension $d$ and dimension $d$ are equivalent for $d \in\{1,2\}$.

Proof. If a poset $P=(X, \leqslant)$ has local dimension 1 , then a local realizer of $P$ of width 1 must consist of a single full linear order on $X$, because antisymmetry of $\leqslant$ requires that every pair $x, y \in X$ occurs in at least one partial linear extension.

Now, let $P=(X, \leqslant)$ be a poset with local dimension 2 , and consider a local realizer of $P$ of width 2. If $x, y \in X$ are incomparable in $\leqslant$, then both occurrences of $x$ and $y$ are in the same two partial linear extensions, where $x<y$ in one and $x>y$ in the other. Therefore, the subposet of $P$ induced on every connected component $C$ of the incomparability graph of $P$ is witnessed by two partial linear extensions, which restricted to $C$ form a realizer of that subposet. These realizers stacked according to the order $\leqslant$ form a realizer of $P$ of size 2 .

C. The standard examples $S_{k}$ have boolean dimension 4 when $k \geqslant 4$, local dimension 3 when $k \geqslant 3$, and dimension $k$ when $k \geqslant 2$.

Proof. It was observed in [10] that the standard example $S_{k}$ has boolean dimension 4 (when $k \geqslant 4)$, witnessed by the formula $\phi(\alpha)=\alpha_{1} \wedge \alpha_{2} \wedge\left(\alpha_{3} \vee \alpha_{4}\right)$ and the following four linear orders:

$$
\begin{array}{ll}
a_{1}<\cdots<a_{k}<b_{1}<\cdots<b_{k}, & b_{1}<a_{1}<\cdots<b_{k}<a_{k}, \\
a_{k}<\cdots<a_{1}<b_{k}<\cdots<b_{1}, & b_{k}<a_{k}<\cdots<b_{1}<a_{1} .
\end{array}
$$

Ueckerdt [26] observed that $S_{k}$ has local dimension 3 (when $k \geqslant 3$ ), witnessed by the two linear extensions above on the left and $k$ partial linear extensions each of the form $b_{i}<a_{i}$. 
Only one pair $a_{i}, b_{i}$ can be ordered as $b_{i}<a_{i}$ in a single linear extension, so the dimension of $S_{k}$ is at least $k$. A realizer of size exactly $k$ can be constructed easily when $k \geqslant 2$, see [8].

D. There are posets with boolean dimension 4 and unbounded local dimension.

Proof. Another well-known construction of posets with arbitrarily large dimension involves incidence posets of complete graphs: $P_{n}=(V \cup E, \leqslant)$, where $V=\left\{v_{1}, \ldots, v_{n}\right\}$ are the minimal elements, $E=\left\{v_{1} v_{2}, v_{1} v_{3}, \ldots, v_{n-1} v_{n}\right\}$ are the maximal elements, and the only comparable pairs are $v_{i}<v_{i} v_{j}$ and $v_{j}<v_{i} v_{j}$ for $i \neq j$. The dimension of $P_{n}$ is at least $\log _{2} \log _{2} n$ [8, Theorem 4.22]. The boolean dimension of $P_{n}$ is at most 4 , witnessed by the formula $\phi(\alpha)=\left(\alpha_{1} \wedge \alpha_{2}\right) \vee\left(\alpha_{3} \wedge \alpha_{4}\right)$ and the following four linear orders:

$$
\begin{aligned}
& A_{1}<\cdots<A_{n} \text {, where each } A_{i} \text { has form } v_{i}<v_{i} v_{i+1}<\cdots<v_{i} v_{n}, \\
& B_{n}<\cdots<B_{1} \text {, where each } B_{i} \text { has form } v_{i}<v_{i} v_{n}<\cdots<v_{i} v_{i+1}, \\
& C_{1}<\cdots<C_{n} \text {, where each } C_{i} \text { has form } v_{i}<v_{1} v_{i}<\cdots<v_{i-1} v_{i}, \\
& D_{n}<\cdots<D_{1} \text {, where each } D_{i} \text { has form } v_{i}<v_{i-1} v_{i}<\cdots<v_{1} v_{i} .
\end{aligned}
$$

The local dimension of $P_{n}$ is unbounded as $n \rightarrow \infty$. For suppose $P_{n}$ has a local realizer of width $d$. Enumerate the occurrences of each element of $V \cup E$ in the local realizer from 1 to (at most) $d$. Each triple $v_{i} v_{j} v_{k}(i<j<k)$ can be assigned a color $(p, q)$ so that $v_{i} v_{k}<v_{j}$ in a partial linear extension containing the $p$ th occurrence of $v_{j}$ and the $q$ th occurrence of $v_{i} v_{k}$. By Ramsey's theorem, if $n$ is large enough compared to $d$, then there is a quadruple $v_{i} v_{j} v_{k} v_{\ell}$ $(i<j<k<\ell)$ with all four triples of the same color $(p, q)$. It follows that the $p$ th occurrences of $v_{j}$ and $v_{k}$ and the $q$ th occurrences of $v_{i} v_{\ell}, v_{i} v_{k}$, and $v_{j} v_{\ell}$ are all in the same partial linear extension, which therefore contains a cycle $v_{j}<v_{j} v_{\ell}<v_{k}<v_{i} v_{k}<v_{j}$, a contradiction.

\section{E. Posets with local dimension 3 have bounded boolean dimension.}

Proof. Let $P=(X, \leqslant)$ be a poset with a local realizer of width 3 consisting of partial linear extensions that we call gadgets. We construct a boolean realizer $\left\{\leqslant^{\star}, \leqslant_{1}, \leqslant_{1}^{\prime}, \ldots, \leqslant_{d}, \leqslant_{d}^{\prime}\right\}$ for a formula of the form $\alpha^{\star} \wedge\left(\alpha_{1} \vee \alpha_{1}^{\prime}\right) \wedge \cdots \wedge\left(\alpha_{d} \vee \alpha_{d}^{\prime}\right)$. The order $\leqslant^{\star}$ is an arbitrary linear extension of $\leqslant$ on $X$. Each pair of orders $\leqslant_{i}, \leqslant_{i}^{\prime}$ is defined by $X_{1}<_{i} \cdots<_{i} X_{t}$ and $X_{t}<_{i}^{\prime} \cdots<_{i}^{\prime} X_{1}$, where $\left\{X_{1}, \ldots, X_{t}\right\}$ is some partition of $X$ into blocks such that every block $X_{j}$ is completely ordered by some gadget and that order is inherited by $\leqslant_{i}$ and $\leqslant_{i}^{\prime}$. Then, we have $x<y$ for the relation $\leqslant$ defined by (1) if and only if $x<^{\star} y$ and $x<y$ in every block containing both $x$ and $y$. It remains to construct a bounded number of partitions of $X$ into blocks so that for any $x, y \in X$, if $x<^{\star} y$ and $x>y$ in some gadget, then $x>y$ in some block in at least one of the partitions.

Without loss of generality, assume that each element $x \in X$ has exactly 3 occurrences in the gadgets - enumerate them as $x^{1}, x^{2}, x^{3}$ according to a fixed order of the gadgets. For each $p \in\{1,2,3\}$, form a partition of $X$ by restricting every gadget to elements of the form $x^{p}$. These three partitions witness all comparabilities of the form $x^{p}>y^{p}$ within gadgets.

Now, let $G$ be a graph on $X$ where $x y$ (with $x<^{\star} y$ ) is an edge if and only if $x^{p}>y^{q}(p \neq q)$ in some gadget. Thus $G$ is a subgraph of the incomparability graph of $P$. Suppose $\chi(G)>38$. It follows that $G$ has an edge $u v$ such that $\chi\left(G\left[X_{u v}\right]\right) \geqslant 19$, where $X_{u v}=\left\{x \in X: u<^{\star} x<^{\star} v\right\}[20$, Lemma 2.1]. Let $X_{u}=\left\{x \in X_{u v}: u \nless x\right\}$ and $X_{v}=\left\{x \in X_{u v}: x \nless v\right\}$. Thus $X_{u v}=X_{u} \cup X_{v}$, as $u \notin v$. Let a color of $x \in X_{u}$ be a quadruple $(p, q, r, s)$ with $p<q$ and $r<s$, where either $x^{p}<u^{q}$ and $x^{r}>u^{s}$ or $x^{p}>u^{q}$ and $x^{r}<u^{s}$ in some gadgets. There are 9 possible colors (quadruples). The coloring of $G\left[X_{u}\right]$ thus obtained is proper-whenever $x, y \in X_{u}$ have the same color, $x^{p}$ and $y^{p}$ are in the same gadget, as well as $x^{r}$ and $y^{r}$ are in the same gadget; this contradicts the fact that the edge $x y$ of $G$ is witnessed by some $x^{i}$ and $y^{j}$ with $i \neq j$ occurring in the same gadget (this is where we use the bound 3 on the number of occurrences). Thus $\chi\left(G\left[X_{u}\right]\right) \leqslant 9$, and similarly $\chi\left(G\left[X_{v}\right]\right) \leqslant 9$, which yields $\chi\left(G\left[X_{u v}\right]\right) \leqslant 18$. This contradiction shows $\chi(G) \leqslant 38$.

Let $c$ be a proper 38-coloring of $G$. For $1 \leqslant p<q \leqslant 3$ and any distinct colors $a, b$, form a partition of $X$ by restricting every gadget to elements of the form $x^{p}$ with $c(x)=a$ and $y^{q}$ with 
$c(y)=b$ (adding singletons if necessary to obtain a full partition of $X$ ). The 4218 partitions thus obtained have the desired property. The resulting boolean realizer of $P$ has size 8443 .

\section{F. There are posets with local dimension 4 and unbounded boolean dimension.}

Proof. When $(V, E)$ is an acyclic digraph, $v \in V$, and $X, Y \subseteq V$, let $E(X, v)=\{x v \in E: x \in X\}$, $E(v, Y)=\{v y \in E: y \in Y\}$, and $E(X, Y)=\{x y \in E: x \in X$ and $y \in Y\}$ (xy denotes a directed edge from $x$ to $y)$. For every $k \geqslant 1$, we construct an acyclic digraph $G=(V, E)$ with $\chi(G)>k$, a poset $P=(E, \leqslant)$, and a local realizer $\left\{\leqslant_{A}, \leqslant_{B}\right\} \cup\left\{\leqslant_{v}: v \in V\right\}$ of $P$ of width 4 , where

(i) $\leqslant{ }_{A}$ is a linear extension of $\leqslant$ on $E$ such that $E(V, v)<_{A} E(v, V)$ for every $v \in V$,

(ii) $\leqslant B$ is a linear extension of $\leqslant$ on $E$ such that $E(V, v)<_{B} E(v, V)$ and $E(v, V)$ occurs as a contiguous block in $\leqslant_{B}$ for every $v \in V$,

(iii) $\leqslant_{v}$ is a gadget - a partial linear extension of the form $E(v, V)<_{v} E(V, v)$ for every $v \in V$.

The construction is an adaptation of the well-known construction of triangle-free graphs with arbitrarily large chromatic number from [7, 18]. For $k=1$, let $V=\{u, v\}, E=\{u v\}$, and $\leqslant A, \leqslant_{B}, \leqslant_{u}, \leqslant_{v}$ be trivial orders on $E$. Now, suppose that $k \geqslant 2$ and the construction can be performed for $k-1$. Let $r$ be the number of vertices in that construction, $s=k(r-1)+1$, and $n=\left(\begin{array}{l}s \\ r\end{array}\right)$. For $1 \leqslant i \leqslant n$, let $G^{i}=\left(V^{i}, E^{i}\right), P^{i}=\left(E^{i}, \leqslant i\right)$, and $\left\{\leqslant_{A}^{i}, \leqslant_{B}^{i}\right\} \cup\left\{\leqslant \leqslant_{v}^{i}: v \in V^{i}\right\}$ be separate instances of the construction for $k-1$. Let $X=\left\{x_{1}, \ldots, x_{s}\right\}$ be yet a separate set of $s$ vertices. Let $X^{1}, \ldots, X^{n}$ be the $r$-element subsets of $X$. Let $V=X \cup V^{1} \cup \cdots \cup V^{n}$ and $E=\bigcup_{i=1}^{n}\left(\left\{x_{1}^{i} v_{1}^{i}, \ldots, x_{r}^{i} v_{r}^{i}\right\} \cup E^{i}\right)$, where

- $x_{1}^{i}, \ldots, x_{r}^{i}$ are the vertices in $X^{i}$ in the same order as in the sequence $x_{1}, \ldots, x_{s}$,

- $v_{1}^{i}, \ldots, v_{r}^{i}$ are the vertices in $V^{i}$ ordered so that $E^{i}\left(v_{1}^{i}, V^{i}\right)<_{B}^{i} \cdots<_{B}^{i} E^{i}\left(v_{r}^{i}, V^{i}\right)$.

Let $G=(V, E)$. Clearly, $G$ is an acyclic digraph. The assumption that $\chi\left(G^{i}\right)>k-1$ for all $i$ implies $\chi(G)>k[7,18]$. Indeed, in any proper $k$-coloring of $G$, at least one of the sets $X^{i}$ would be monochromatic, which would yield $\chi\left(G^{i}\right) \leqslant k-1$, a contradiction. For $1 \leqslant j \leqslant s$, let $N_{j}=\left\{v \in V: x_{j} v \in E\right\}$. Let $\leqslant_{A}$ and $\leqslant_{B}$ be linear orders on $V$ such that

- $E\left(X^{1}, V^{1}\right)<_{A} E^{1}<_{A} \cdots<_{A} E\left(X^{n}, V^{n}\right)<_{A} E^{n}$ and the restriction of $\leqslant_{A}$ to each $E^{i}$ is $\leqslant_{A}^{i}$,

- $E\left(x_{1}, V\right)<_{B} E\left(N_{1}, V\right)<_{B} \cdots<_{B} E\left(x_{s}, V\right)<_{B} E\left(N_{s}, V\right)$.

The latter property implies that the restriction of $\leqslant_{B}$ to each $E^{i}$ is $\leqslant_{B}^{i}$. Finally, for every $x \in X$, let $\leqslant x$ be a new gadget on $E(x, V)$, and for $v \in V^{i}$ and $1 \leqslant i \leqslant n$, let $\leqslant v$ be $\leqslant_{v}^{i}$ with $E(X, v)$ (which is just one edge) added on top. This guarantees properties (i)-(iii). Let $\leqslant$ be the relation on $E$ defined from $\left\{\leqslant_{A}, \leqslant_{B}\right\} \cup\left\{\leqslant_{v}: v \in V\right\}$ by (2). It follows that the restriction of $\leqslant$ to each $E^{i}$ is $\leqslant i$. It remains to show that the relation $\leqslant$ is a partial order, so that $P=(E, \leqslant)$ is a poset and $\left\{\leqslant_{A}, \leqslant_{B}\right\} \cup\left\{\leqslant_{v}: v \in V\right\}$ is its local realizer.

Reflexivity and antisymmetry of $\leqslant$ are clear. For transitivity, suppose $e, f, g \in E, e<f$, and $f<g$, but $e \notin g$. The assumption that $e<f$ and $f<g$ implies $e<_{A} f<_{A} g$ and $e<_{B} f<_{B} g$. Since $e \notin g$, the edges $e$ and $g$ must occur as $e>g$ in some gadget. We consider four cases.

1. If $e, g \in E^{i}$ for some $i$, then the definition of $\leqslant A$ implies $f \in E^{i}$, so $e<^{i} f<^{i} g$. This and the assumption that $e \notin g$ contradict the fact that $\leqslant i$ is the restriction of $\leqslant$ to $E^{i}$.

2. If $e, g \in E(x, V)$ for some $x \in X$, then the definition of $\leqslant B$ implies $f \in E(x, V)$. This yields $e<_{x} f<_{x} g$, and the only gadget containing both $e$ and $g$ fails to witness $e \notin g$.

3. If $e=x_{j} v$ and $g=u v$ for some $x_{j} \in X$ and $u, v \in V \backslash X$, then $u, v \in V^{i}$ for some $i$. The definition of $E$ implies that there is an edge $x_{j^{\prime}} u \in E$, where $j^{\prime}<j$, and therefore $g \in E\left(N_{j^{\prime}}, V\right)<_{B} E\left(x_{j}, V\right) \ni e$, a contradiction.

4. If $e=x_{j} v$ and $g=v w$ for some $x_{j} \in X$ and $v, w \in V \backslash X$, then $v, w \in V^{i}$ for some $i$. The definitions of $\leqslant_{A}$ and $\leqslant_{B}$ imply

$$
f \in\left(E\left(X^{i}, V^{i}\right) \cup E^{i}\right) \cap\left(E\left(x_{j}, V\right) \cup E\left(N_{j}, V\right)\right)=\left\{x_{j} v\right\} \cup E\left(v, V^{i}\right) .
$$

This yields $e<_{v} f<_{v} g$, and the only gadget containing both $e$ and $g$ fails to witness $e \nless g$. 
This shows that $\leqslant$ is transitive, thus completing the proof of correctness of the construction.

Let $k=2^{2^{2^{d}}}$. We show that the poset $P$ resulting from the construction above has boolean dimension greater than $d$. For suppose $\left\{\leqslant_{1}, \ldots, \leqslant_{d}\right\}$ is a boolean realizer of $P$ for a formula $\phi$. Let $G^{\prime}=(E, A)$ be the arc digraph of $G$, and let $G^{\prime \prime}=(A, B)$ be the arc digraph of $G^{\prime}$. That is, $A=\{u v w: u v, v w \in E\}$ and $B=\{u v w x: u v w, v w x \in A\}=\{u v w x: u v, v w, w x \in E\}$. It follows that $\chi\left(G^{\prime}\right) \geqslant \log _{2} \chi(G)$ and $\chi\left(G^{\prime \prime}\right) \geqslant \log _{2} \chi\left(G^{\prime}\right)\left[11\right.$, Theorem 9], and thus $\chi\left(G^{\prime \prime}\right)>2^{d}$. For $u v w \in A$, let $\alpha(u v w)=\left(\left(u v<_{1} v w\right), \ldots,\left(u v<_{d} v w\right)\right) \in\{0,1\}^{d}$; the fact that $u v>_{v} v w$ implies $u v \notin v w$ and thus $\phi(\alpha(u v w))=0$. Let $u v w x \in B$. We have $u v<_{A} v w<_{A} w x$ and $u v<_{B} v w<_{B} w x$, which implies $u v<w x$, because no gadget contains both $u v$ and $w x$. If $\alpha(u v w)=\alpha(v w x)=\alpha$, then transitivity of $\leqslant_{1}, \ldots, \leqslant_{d}$ implies $\left(\left(u v<_{1} w x\right), \ldots,\left(u v<_{d} w x\right)\right)=$ $\alpha$. This, $\phi(\alpha)=0$, and $u v<w x$ result in a contradiction. Therefore, $\alpha: A \rightarrow\{0,1\}^{d}$ is a proper $2^{d}$-coloring of $G^{\prime \prime}$. This contradicts the fact that $\chi\left(G^{\prime \prime}\right)>2^{d}$.

\section{REFERENCES}

[1] Kirby A. Baker, Peter C. Fishburn, and Fred S. Roberts, Partial orders of dimension 2, Networks 2 (1), $11-28,1972$

[2] Fidel Barrera Cruz, Thomas Prag, Heather C. Smith, Libby Taylor, and William T. Trotter, Local dimension, dimension and topological graph theory, manuscript.

[3] Csaba Biró, Mitchel T. Keller, and Stephen J. Young, Posets with cover graph of pathwidth two have bounded dimension, Order 33 (2), 195-212, 2016.

[4] Thomas Bläsius, Peter Stumpf, and Torsten Ueckerdt, Local and union boxicity, arXiv:1609.09447.

[5] Bartłomiej Bosek, Jarosław Grytczuk, and William T. Trotter, Local dimension is unbounded for planar posets, manuscript.

[6] Graham R. Brightwell and Paolo G. Franciosa, On the Boolean dimension of spherical orders, Order 13 (3), 233-243, 1996.

[7] Blanche Descartes, Solution to advanced problem 4526, Amer. Math. Monthly 61 (5), 352-353, 1954.

[8] Ben Dushnik and Edwin W. Miller, Partially ordered sets, Amer. J. Math. 63 (3), 600-610, 1941.

[9] Stefan Felsner, Ching Man Li, and William T. Trotter, Adjacency posets of planar graphs, Discrete Math. 310 (5), 1097-1104, 2010.

[10] Giorgio Gambosi, Jaroslav Nešetřil, and Maurizio Talamo, On locally presented posets, Theor. Comput. Sci. $70(2), 251-260,1990$.

[11] Charles C. Harner and Roger C. Entringer, Arc colorings of digraphs, J. Combin. Theory Ser. B 13 (3), 219-225, 1972.

[12] Toshio Hiraguchi, On the dimension of partially ordered sets, Sci. Rep. Kanazawa Univ. 1 (2), 77-94, 1951.

[13] David M. Howard, Noah Streib, William T. Trotter, Bartosz Walczak, and Ruidong Wang, The dimension of posets with planar cover graphs excluding two long incomparable chains, arXiv:1608.08843.

[14] Gwenaël Joret, Piotr Micek, Kevin G. Milans, William T. Trotter, Bartosz Walczak, and Ruidong Wang, Tree-width and dimension, Combinatorica 36 (4), 431-450, 2016.

[15] Gwenaël Joret, Piotr Micek, William T. Trotter, Ruidong Wang, and Veit Wiechert, On the dimension of posets with cover graphs of treewidth 2 , Order, in press.

[16] Gwenaël Joret, Piotr Micek, and Veit Wiechert, Sparsity and dimension, in: 27th Annual ACM-SIAM Symposium on Discrete Algorithms (SODA 2016), pp. 1804-1813, SIAM, Philadelphia, 2016.

[17] David Kelly, On the dimension of partially ordered sets, Discrete Math. 35 (1-3), 135-156, 1981.

[18] John B. Kelly and Le Roy M. Kelly, Path and circuits in critical graphs, Amer. J. Math. 76 (4), 786-792, 1954.

[19] Kolja Knauer and Torsten Ueckerdt, Three ways to cover a graph, Discrete Math. 339 (2), 745-758, 2016.

[20] Sean McGuinness, On bounding the chromatic number of L-graphs, Discrete Math. 154 (1-3), 179-187, 1996.

[21] Piotr Micek and Bartosz Walczak, unpublished result.

[22] Jaroslav Nešetřil and Pavel Pudlák, A note on boolean dimension of posets, in: Gábor Halász and Vera T. Sós (eds.), Irregularities of Partitions, vol. 8 of Algorithms Combin., pp. 137-140, Springer, Berlin, 1989.

[23] Noah Streib and William T. Trotter, Dimension and height for posets with planar cover graphs, European J. Combin. 35, 474-489, 2014.

[24] William T. Trotter, Order preserving embeddings of aographs, in: Yousef Alavi and Don R. Lick (eds.), Theory and Applications of Graphs, vol. 642 of Lecture Notes Math., pp. 572-579, Springer, Berlin, 1978.

[25] William T. Trotter and John I. Moore, The dimension of planar posets, J. Combin. Theory Ser. B 22 (1), 54-67, 1977.

[26] Torsten Ueckerdt, Order \& Geometry Workshop, Gultowy, 2016.

[27] Bartosz Walczak, Minors and dimension, J. Combin. Theory Ser. B 122, 668-689, 2017. 